\title{
Serial computed tomographic evaluation in desquamative interstitial pneumonia
}

\author{
Masanori Akira, Satoru Yamamoto, Hideki Hara, Mitsunori Sakatani, Einosuke Ueda
}

\begin{abstract}
Background - Desquamative interstitial pneumonia (DIP) may represent the early stage and usual interstitial pneumonia (UIP) the late stage of the same disease. The purpose of this study was to evaluate the computed tomographic (CT) features of DIP, to evaluate the changes in pattern and extent of disease over time, and to determine whether the appearances of DIP on the CT scan change to those of UIP during follow up.

Methods - Sequential CT evaluation was conducted on eight patients with DIP over a mean (SD) follow up period of 3.2 (1.3) years (range 1.6-6.5). The relative extents of ground glass and honeycombing were determined from serial CT scans. Changes in the extent and appearance of the disease were examined in paired anatomically comparable CT sections.
\end{abstract}

Results - Common features on the CT scans of patients with DIP were a homogeneous increase in lung attenuation $(n=$ $5)$, linear areas of attenuation $(n=5)$, relatively well preserved lung architecture $(n=5)$, and the presence of small cysts $(n=$ 6). Uncommon features were architectural distortion $(n=3)$ and traction bronchiectasis $(n=1)$. In six patients with DIP with cystic spaces these did not change with time in three cases, in two they regressed, and in one patient they increased. Open lung biopsy samples from patients with DIP with many cystic lesions showed dilated alveolar ducts and bronchioles and/ or pulmonary cysts, as well as numerous macrophage-filled air spaces and mild fibrosis, but no typical honeycomb cysts were seen.

Conclusions - Some of the microcysts in DIP are different from the honeycomb cysts seen in UIP, and some of the cysts seen in patients with DIP resolve with time. DIP does not progress to UIP in the short term.

(Thorax 1997;52:333-337)

Keywords: computed tomography, desquamative interstitial pneumonia, usual interstitial pneumonia.

National Kinki Chuo Hospital for Chest Disease, 1180

Nagasone-cho, Sakai City, Osaka 591, Japan

Correspondence to: Dr M Akira.

Received 30 August 1996 Returned to authors 16 September 1996

Revised version received

18 October 1996

Accepted for publication

16 January 1997

Desquamative interstitial pneumonia (DIP) was first described by Liebow et $a l^{1}$ in 1965 . Carrington et $a l^{2}$ showed that desquamative interstitial pneumonia and usual interstitial pneumonia (UIP) are two distinct lesions that differ in their history and their response to steroid therapy. Thus, patients with DIP have a better prognosis and a better response to treatment with corticosteroids than patients with UIP. However, other observers ${ }^{34}$ have suggested that DIP may represent an early stage of idiopathic pulmonary fibrosis which subsequently progresses to UIP. The morphology of both may appear in the same patient at the same time, and although in one study patients with DIP treated with corticosteroids were found ultimately to develop extensive fibrosis and honeycombing, ${ }^{5}$ others ${ }^{67}$ have suggested that DIP does not necessarily progress to UIP and can relapse many years after the initial presentation with a similar histological picture.

Computed tomographic (CT) scanning has an established role in the evaluation of idiopathic pulmonary fibrosis. ${ }^{89}$ On the CT scan DIP is characterised by patchy areas of ground glass opacification, predominantly on the periphery of the lung. ${ }^{10}$ It has recently been suggested that the greater extent of ground glass attenuation and the paucity of cystic changes in patients with DIP should enable it to be distinguished from UIP. ${ }^{11}$

UIP is easily distinguished from DIP by the presence of honeycomb cysts. ${ }^{10}$ However, cystic changes may also be seen but are less prominent in DIP. ${ }^{11}$ DIP should change to UIP during follow up if DIP represents the early stage and UIP the late stage of the same disease.

The purpose of this study was to assess the CT features of DIP, to evaluate the changes of the pattern and extent of the disease with time, and to determine whether the CT appearances of DIP change to those of UIP during follow up.

\section{Methods}

PATIENTS

All patients followed at our hospital from January 1985 to September 1996 with a diagnosis of DIP who had undergone serial thoracic CT scanning were included. None had evidence of a connective tissue disorder, hypersensitivity pneumonitis, left ventricular dysfunction, cardiac valvular disease, or significant occupational exposure. Eight patients (seven men) of mean (SD) age 56.0 (7.7) at the first CT scan were studied. Six were heavy smokers ( $\geq 25$ pack years), one had light smoking history ( $\leq 10$ pack years), and one did not smoke.
The diagnosis of DIP was based on histologica criteria from open lung biopsy samples in all patients and included the presence of numerous macrophages filling the air spaces, relatively mild fibrosis, and uniformity of the histological features from field to field. The time from the initial CT scan to biopsy was less than a month 
in all patients. Biopsy samples were obtained from two or three different lobes, with one specimen per lobe in all cases. The biopsy specimens were studied by several pathologists. All patients were symptomatic, but none had received corticosteroids before the initial examination. The patients were treated between the CT scans with prednisolone at an initial daily dose of 40-60 mg.

CT PROTOCOL

Scanning was performed with a GE 8800 (GE Medical Systems, Milwaukee, USA) or Quantex Plus (Yokogawa Medical Systems, Hino, Japan) CT unit. CT scans of sections $1.5-2.0 \mathrm{~mm}$ in thickness were taken at $20 \mathrm{~mm}$ intervals from the lung apices to below the costophrenic angle at full inspiration with the patient in the supine position. Prone views were not used. All images were reconstructed with a high resolution algorithm. Window levels were set for optimal imaging of the lungs (level, $-700 \mathrm{HU}$; width, $1200 \mathrm{HU})$. Scout views
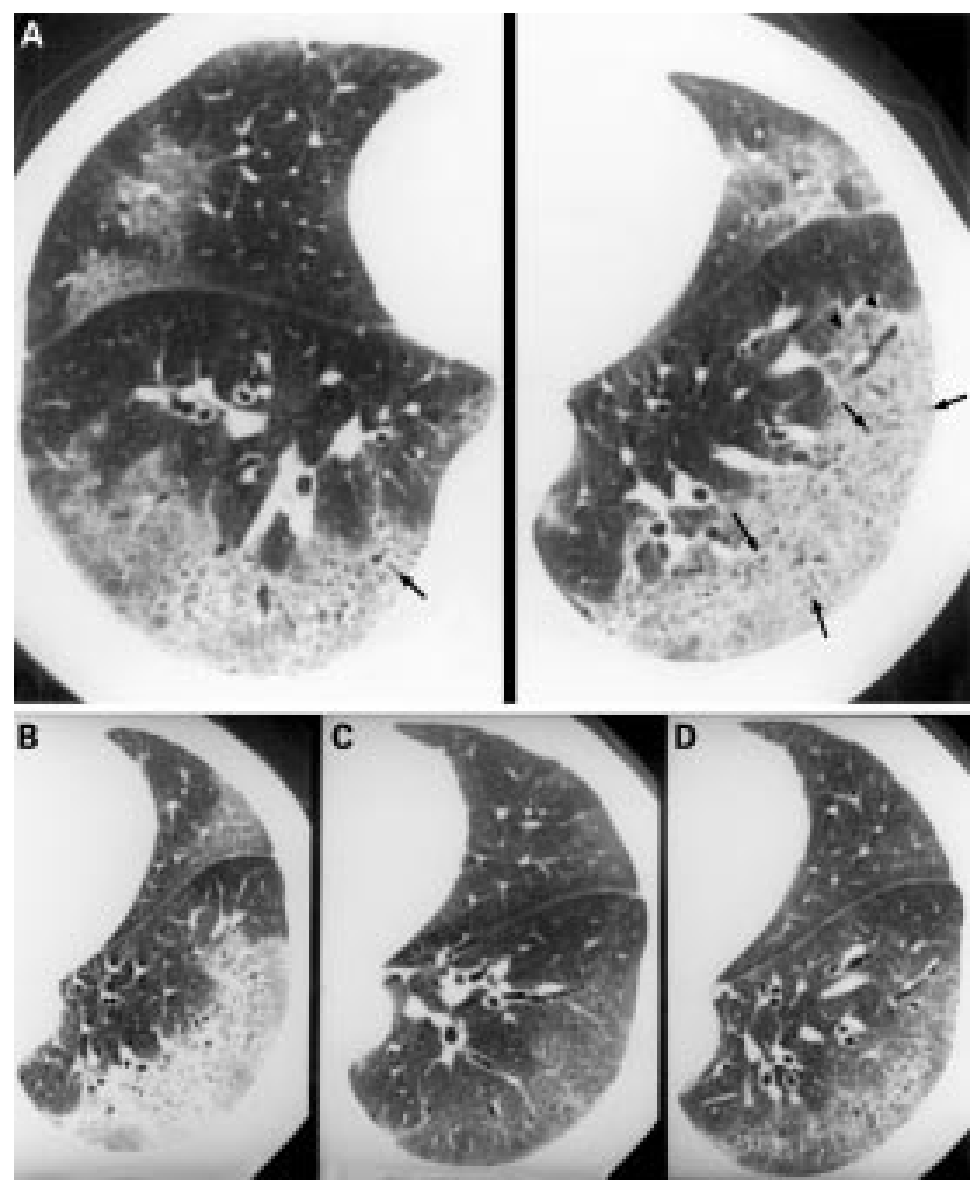

Figure 1 Serial CT scans from a 60 year old man with desquamative interstitial pneumonia (DIP). (A) HRCT scan through the lower lung zone showing diffuse bilateral ground glass areas of attenuation. Very few small cystic spaces are present (small arrows), most of which may be cross sections of dilated bronchioles. A dilated air bronchiologram showing a Y-shaped tubular structure can be seen within the subpleural ground glass areas of attenuation (large arrows), some of which expand at the end of the branching structure. The dilated air bronchogram is smooth (arrowheads). (B), (C), and (D) CT scans of sections through the lower part of the left lung obtained at two months, 1.5 years, and 2.5 years, respectively, after diagnosis. (B) shows the increase in ground glass attenuation before treatment, (C) shows the considerable improvement in ground glass attenuation seen after corticosteroid treatment, and in (D) a slight increase in ground glass attenuation is seen 2.5 years later. provided anatomical references to ensure that similar planes were obtained in serial CT studies. The CT scans were obtained as part of a prospective protocol to evaluate the progress of the disease and were made at the time of the initial visit to hospital and then one month after treatment with corticosteroids. Follow up CT scans were obtained at 6-12 months and final follow up scans at 1.6-6.5 years after the initial CT examination (mean 3.2 years).

CT GRADING SYSTEM

CT scans were evaluated independently and randomly by two chest radiologists who had no knowledge of the clinical and pathological data, and the final assessment was by consensus. The dates of the scans were concealed and sections were scored in random order. Assessment was made of the presence and extent of ground glass attenuated areas and cystic spaces. The former were defined as those of hazy increased attenuation not obscuring underlying vascular markings. For each CT section the extent of the disease was scored for ground glass attenuation and honeycombing. All these parameters were visually estimated from percentages of lung parenchyma with abnormal appearance to the nearest $5 \%$ of parenchymal involvement. Scores for each section were averaged to obtain one average score, using a weighting factor to make allowances for differences in lung volumes at the different levels. The ratio of the volumes of the upper, middle, and lower lung zones (1.0:1.6:1.3) was used as the correction factor for lung volume as described by Müller et al. ${ }^{12}$

In addition, the following $\mathrm{CT}$ features within areas of lung hyperattenuation were also noted: (a) homogeneous or heterogeneous increase in lung attenuation; (b) relatively well preserved morphological structures of underlying parenchyma or architectural distortion occurring when secondary pulmonary lobules, bronchi, and vessels were distorted; (c) traction bronchiectasis and bronchiolectasis; and (d) linear areas of attenuation including septal and nonseptal lines.

\section{PULMONARY FUNCTION TESTS}

Pulmonary function tests were performed within one month of the CT scans. Forced vital capacity (FVC) and forced expiratory volume in one second $\left(\mathrm{FEV}_{1}\right)$ were determined with an Autospirometer (Minato, Osaka, Japan). Carbon monoxide transfer factor (TLCO) was measured by the single breath method and corrected for alveolar volume and haemoglobin level. The data were expressed as percentages of the predicted values.

\section{Results}

APPEARANCES ON INITIAL CT SCAN

The duration of disease from onset to the initial CT examination and the physiological measurements in the patients with DIP are 
Table 1 Mean (SD) clinical data from initial evaluation of eight patients with desquamative interstitial pneumonia (DIP)

\begin{tabular}{ll}
\hline Age (years) & $56(7.7)$ \\
M:F & $7: 1$ \\
Duration of symptoms (months) & $17.0(13.4)$ \\
Initial FVC (\% pred) & $93.2(18.5)$ \\
Initial FEV $/$ FVC (\% pred) & $80.7(4.6)$ \\
Initial TLCO (\% pred) & $52.1(5.8)$ \\
Duration of follow up (years) & $3.2(1.3)$ \\
\hline
\end{tabular}

$\mathrm{FVC}=$ forced vital capacity; $\mathrm{FEV}_{1}=$ forced expiratory volume in one second; TLCO $=$ carbon monoxide transfer factor.

shown in table 1 . The mean (SD) extent of the disease in these patients was $30(16.5) \%$. The
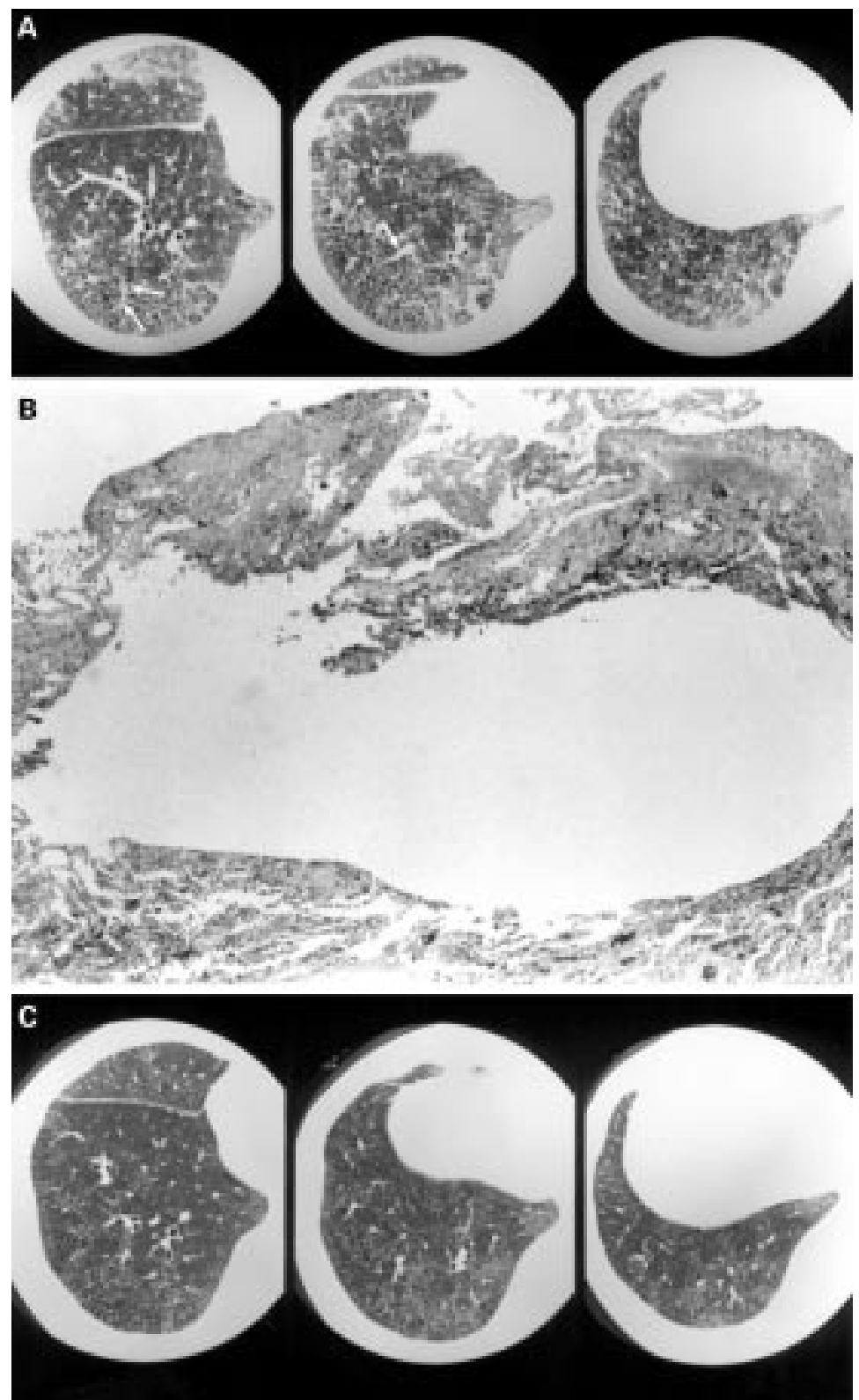

Figure 2 Serial CT scans from a 48 year old man with DIP. (A) CT scans of sections through lower parts of the right lung showing areas of ground glass attenuation with more numerous cystic spaces within the subpleural areas of ground glass attenuation. Although the cystic lesions are numerous, there is only mild distortion of the architecture and pulmonary vessels through the cystic lesions are still preserved and not distorted (arrows). (B) Open lung biopsy specimen of this patient showing dilated bronchioles with the surrounding air spaces filled by numerous macrophages (stain: haematoxylin and eosin; original magnification $\times 12.5)$. (C) CT scans of sections through the lower parts of the right lung at three year intervals showing markedly less ground glass attenuation. Small cystic lesions can be seen but the pulmonary vessels through the cystic lesions are preserved. appearances of DIP on the initial HRCT scan showed ground glass opacity and small cystic lesions (figs 1-3) in the middle and lower lung zones of all patients, predominantly on the periphery of the lung. Areas of ground glass attenuation were present in all eight patients. Other lung changes within the ground glass attenuated areas of these patients were a homogeneous $(n=5)$ or heterogeneous $(n=3)$ increase in lung attenuation, linear areas of attenuation $(\mathrm{n}=5)$, traction bronchiectasis $(\mathrm{n}=1)$, and architectural distortion $(\mathrm{n}=3)$ (table 2). Bronchial dilatation was evident in three patients with DIP, characterised by smooth dilatation (fig 1A), and bronchiolectasis was seen in four cases (fig 1A).

Cystic spaces could be seen in six of the eight patients with DIP. Although cystic lesions were present, the lung architecture appeared to be relatively well preserved in the cystic lesions of four cases with pulmonary vessels passing through them (fig 2). In five cases more than $5 \%$ of the cystic lesions were found on the CT scan (mean 7.5\%; range 5-20\%). However, open lung biopsy samples showed no typical honeycomb cysts in any of the patients with DIP, even when the site of open lung biopsy, which was ascertained from the surgical report and the location of surgical staples, included findings of cystic lesions on the CT scan. In patients with DIP who had a large number of cystic lesions open lung biopsy samples showed dilated alveolar ducts and bronchioles and/or pulmonary cysts, as well as numerous macrophages in the air spaces and mild fibrosis (fig 2B).

FOLLOW UP CT SCAN

The patients with DIP showed a subpleural distribution on the follow up CT scan. A decrease in ground glass opacity was seen in all cases after treatment with corticosteroids (figs $1-3)$, but in three cases the ground glass opacity increased again despite continuous treatment with low dose corticosteroids (fig 1). Three of the six patients with DIP showed no change in the extent of cystic spaces and in two cases the cystic spaces regressed (fig 3). In one of the eight patients with DIP the extent of the cystic spaces increased; this case showed ground glass opacity with heterogeneous and architectural distortion on the initial CT scan, with an increase in the cystic spaces and development of traction bronchiectasis. The patient died of lung cancer and necropsy disclosed interstitial fibrosis and honeycombing. Except for this case, increased attenuation in the patients with DIP was not the same as for those with UIP with time.

\section{Discussion}

The predominant findings on high resolution CT scans in patients with DIP are ground glass areas of attenuation which usually affect the lower lung and subpleural regions. ${ }^{1011}$ These, together with the few cystic changes seen in patients with DIP, have been found to be the features which distinguish DIP from UIP and 

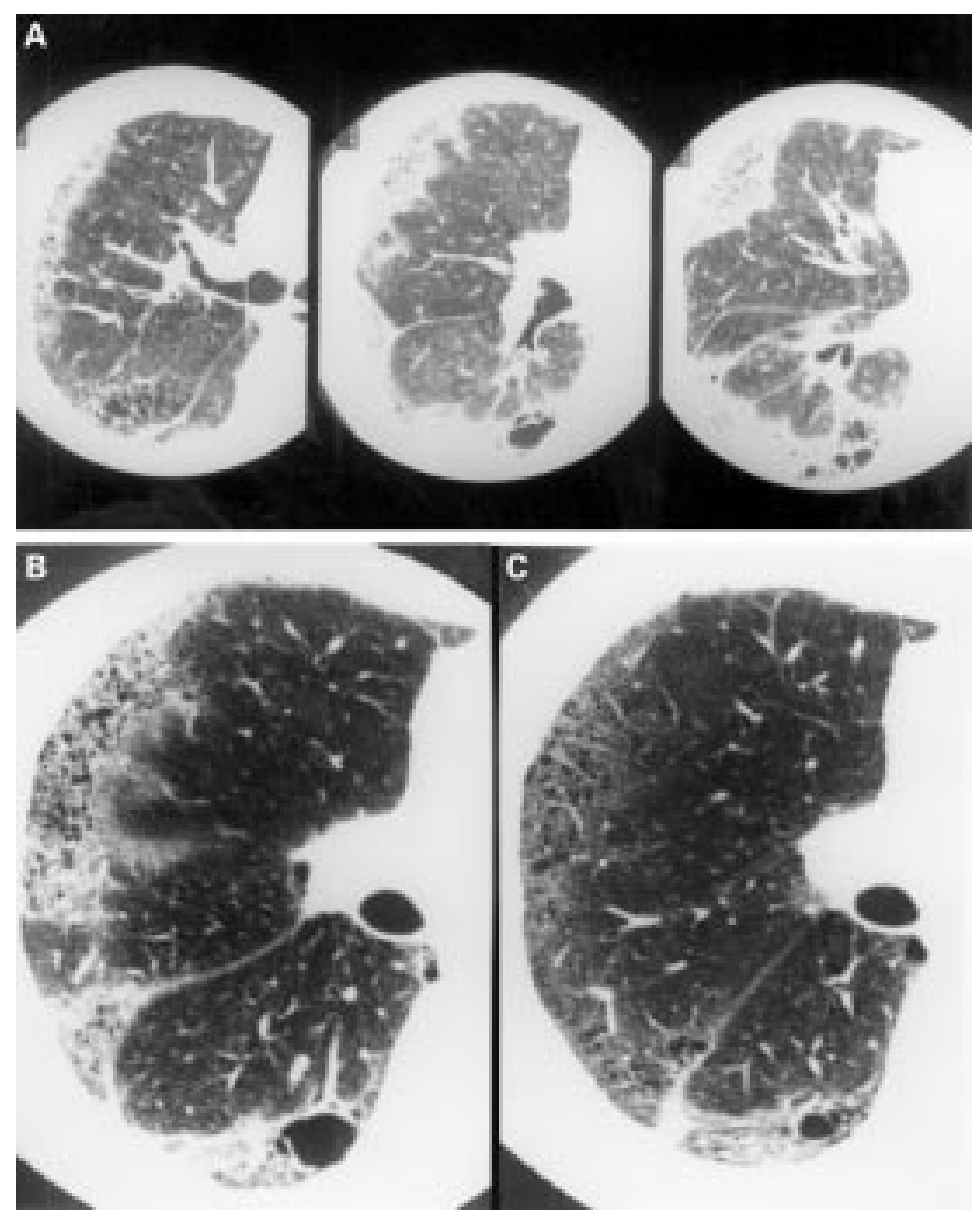

Figure 3 Serial CT scans from a 57 year old man with DIP. (A) CT scans of sections through upper, middle, and lower parts of the right lung showing attenuated ground glass areas at subpleural sites. Small cystic spaces are present within these areas. $(B)$ and $(C)$ $C T$ scans of sections of the right lung at the intermediate bronchus taken two and three years, respectively, after diagnosis showing markedly less ground glass attenuation and more cystic spaces in (B), followed by regression of cystic spaces one year later $(C)$. Similar changes were also seen in cephalic and caudal sections.

Table 2 Computed tomographic (CT) findings from initial and follow up evaluation of eight patients with desquamative pneumonia (DIP)

\begin{tabular}{lll}
\hline CT findings & Initial CT scan & Follow up CT scan \\
\hline Cystic lesions & 5 & 5 \\
Linear areas of attenuation & 5 & 5 \\
Architectural distortion & 3 & 3 \\
Traction bronchiectasis & 1 & 2 \\
Homogeneous/heterogeneous increase in lung attenuation & $5 / 3$ & $5 / 3$ \\
\hline
\end{tabular}

are thus a basis for distinguishing between the two conditions in most cases. ${ }^{11}$ The relatively good preservation of morphological structures of the underlying parenchyma in such areas is commonly seen on the HRCT scan of patients with DIP but rarely in those with UIP. Architectural distortion and traction bronchiectasis and bronchiolectasis are occasionally seen in cases of UIP but are rare in DIP (fig 4). Bronchiectasis in DIP consists of smooth and reversible bronchial dilatation - that is, pseudobronchiectasis ${ }^{13}$ - while traction bronchiectasis in UIP comprises tortuous bronchial dilatation with a convoluted appearance resembling a string of beads. ${ }^{14}$ In our cases of DIP cystic lesions were often seen on the HRCT scan. Follow up scans showed that

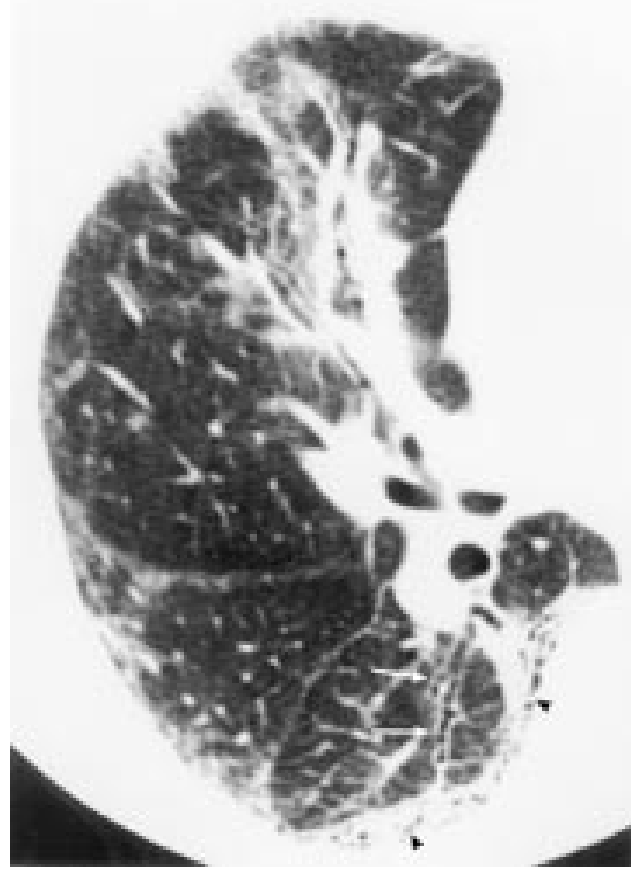

Figure 4 Traction bronchiectasis and bronchiolectasis in a patient with usual interstitial pneumonia (UIP) showing tortuous bronchial dilatation (arrows) and bronchiolectasis within the intensely attenuated lung (arrowheads).

some cystic lesions regressed during treatment, but this was not seen in any patient with UIP despite treatment. ${ }^{1516}$ Honeycomb cysts were not found in the open lung biopsy samples from patients with DIP with many cystic lesions; however, there is variability in lung biopsy samples, even those obtained by open lung biopsy. Open lung biopsy samples showed dilated alveolar ducts and bronchioles as well as numerous macrophages in the air spaces and mild fibrosis. Some of the cystic lesions seen in patients with DIP may therefore be dilated bronchioles or reversible bronchiolectasis.

Ground glass attenuated areas on the CT scans of patients with UIP occur prior to honeycombing and are predictive of it occurring at the same site. ${ }^{1517}$ Progression to honeycombing is seen on the CT scan in most cases of UIP ${ }^{15}$ but only in one of our eight patients with DIP. The initial CT scan of this patient showed a heterogeneous increase in lung attenuation and architectural distortion, both of which features are more common in UIP. Carrington et al reported on five cases with initial histological findings typical of DIP, in whom necroscopic examination showed a combination of the features of fibrosis and intra-alveolar inflammatory changes typical of UIP in one and four had progressed to honeycombing. In the our study most of the patients with DIP showed no progression to honeycombing with time on the CT scan. Hunter and Lamb $^{6}$ noted that patients with DIP relapsed after 12 years of remission and responded to a second course of treatment with steroids. Lipworth et al described two patients diagnosed as having DIP who relapsed seven and 12 years, respectively, after the initial diagnosis. Repeat histological examination of 
open lung biopsy samples taken at the time of relapse showed identical appearances with minimal fibrosis or architectural destruction. ${ }^{7}$ Thus, DIP does not necessarily progress to UIP and can relapse many years after the initial presentation with a similar histological picture.

Our patients had a predominantly subpleural distribution on the initial and follow up CT scans. However, Hartman et $a l^{11}$ found a predominantly subpleural distribution in $59 \%$ of 22 patients with DIP, the distribution in the remaining $41 \%$ being different from that of patients with UIP, most of whom have a predominantly subpleural distribution on CT scanning.

It is concluded that UIP and DIP can be differentiated both initially and on follow up CT scans and that the progression to honeycombing in the two conditions also differs. Some of the cysts in DIP can resolve with time. Serial CT scans may be useful to identify the changes in the lung parenchyma of patients with DIP. This study is limited by the relatively small number of patients. Carefully controlled prospective studies on a larger number of patients are required to comfirm these preliminary results.

1 Liebow AA, Steer A, Billingsley JG. Desquamative interstitial pneumonia. Am $\mathcal{F}$ Med 1965;39:369-404

2 Carrington C, Gaensler EA, Coutu RE, FitzGerald MX, Gupta RG. Natural history and treated course of usual and desquamative interstitial pneumonia. $N$ Engl f Med 1978;298:801-10.
3 Patchefsky AS, Israel HL, Hoch WS, Gordon G. Dequamative interstitial pneumonia: relationship to interstitial fibrosis. Thorax 1973;28:680-93.

4 Tubbs RR, Benjamin SP, Reich NE, McCormack LJ, Scott Van Orstrand H. Desquamative interstitial pneumonia cellular phase of fibrosing alveolitis. Chest 1977;72:15965.

5 McCann BG, Brewer DB. A case of desquamative interstitial pneumonia progressing to "honeycomb lung". F Pathol 1974;112:199-202.

6 Hunter AM, Lamb D. Relapse of fibrosing alveolitis (desquamative interstitial pneumonia) after twelve years. Thorax 1979:34:677-9.

7 Lipworth B, Woodcock A, Addis B, Turner-Warwick M. Late relapse of desquamative interstitial pneumonia. Am Rev Respir Dis 1987;136:1253-5.

8 Müller NL Miller RR. Computed tomography of chronic diffuse infiltrative lung disease: part 1. Am Rev Respir Dis 1990;142:1206-15.

9 Tung KT, Wells AU, Rubens MB, Kirk JME, du Bois RM, Hansell DM. Accuracy of the typical computed RM, Hansell DM. Accuracy of the typical computed tomographic appe

10 Vedal S, Welsh EV, Miller RR, Müller NL. Desquamative interstitial pneumonia: computed tomographic findings interstitial pneumonia: computed tomographic findings 1988;93:215-7.

11 Hartman TE, Primack SL, Swensen SJ, Hansell DM McGuinness G, Müller NL. Desquamative interstitial pneumonia: thin-section CT findings in 22 patients. $R a$ diology 1993;187:787-90

12 Müller NL, Mawson JB, Mathieson JR, Abboud R, Ostrow DN, Champion P. Sarcoidosis: correlation of extent of disease at CT with clinical, functional, and radiographic findings. Radiology 1989;171:613-8.

13 Swensen SJ, Aughenbaugh GL, Douglas WW, Myers JL. High-resolution CT of the lung: findings in various pulmonary diseases. AfR 1992;158:971-9.

14 Westcott JL, Cole SR. Traction bronchiectasis in end-stage pulmonary fibrosis. Radiology 1986;161:665-9.

15 Akira M, Sakatani S, Ueda E. Idiopathic pulmonary fibrosis: progression of honeycombing at thin-section CT. $R a-$ diology 1993;189:687-91.

16 Wells AU, Rubens MB, du Bois RM, Hansell DM. Serial $\mathrm{CT}$ in fibrosing alveolitis: prognostic significance of the initial pattern. AfR 1993;161:1159-65.

17 Terriff BA, Kwan SY, Chan-Yeung MM, Müller NL. Fibrosing alveolitis: chest radiography and $\mathrm{CT}$ as predictors of clinical and functional impairment at follow-up in 26 patients. Radiology 1992;184:445-9. 